\title{
Prognostic value of pre-treatment peripheral blood markers in pancreatic ductal adenocarcinoma and their association with S100A4 expression in tumor tissue
}

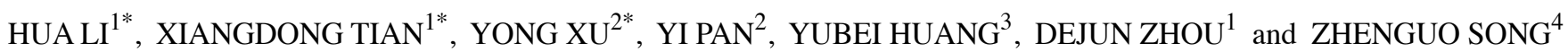 \\ Departments of ${ }^{1}$ Endoscopic Diagnosis and Therapy, ${ }^{2}$ Pathology, ${ }^{3}$ Epidemiological Laboratory and ${ }^{4}$ Anesthesiology, \\ Tianjin Medical University Cancer Institute and Hospital, National Clinical Research Center for Cancer, Key Laboratory \\ of Cancer Prevention and Therapy of Tianjin, Tianjin Clinical Research Center for Cancer, Tianjin 300060, P.R. China
}

Received August 30, 2018; Accepted July 12, 2019

DOI: $10.3892 /$ ol.2019.10809

\begin{abstract}
The aims of the present study were to clarify the prognostic value of peripheral blood variables in patients with pancreatic ductal adenocarcinoma (PDAC), including the neutrophil-to-lymphocyte ratio (NLR), platelet-to-lymphocyte ratio (PLR) and lymphocyte-to-monocyte ratio (LMR), and to determine the association between these variables and S100 calcium-binding protein A4 (S100A4) expression in tumor tissue, which is another prognostic factor for PDAC. Patients with PDAC were recruited at the Tianjin Medical University Cancer Institute and Hospital (Tianjin, China) between December 2008 and December 2014. A retrospective analysis was performed based on the recorded pre-treatment hematological parameters and clinical data. The prognostic value of NLR, PLR and LMR was examined. The association between these variables and S100A4 tissue expression was analyzed. Descriptive statistics and $\chi^{2}$ analyses were used in the present study. The median overall survival (OS) time of patients with PDAC was 9 months
\end{abstract}

Correspondence to: Professor Dejun Zhou, Department of Endoscopic Diagnosis and Therapy, Tianjin Medical University Cancer Institute and Hospital, National Clinical Research Center for Cancer, Key Laboratory of Cancer Prevention and Therapy of Tianjin, Tianjin Clinical Research Center for Cancer, Huanhuxi Road, Hexi, Tianjin 300060, P.R. China

E-mail:632229699@qq.com

Professor Zhenguo Song, Department of Anesthesiology, Tianjin Medical University Cancer Institute and Hospital, National Clinical Research Center for Cancer, Key Laboratory of Cancer Prevention and Therapy of Tianjin, Tianjin Clinical Research Center for Cancer, Huanhuxi Road, Hexi, Tianjin 300060, P.R. China

E-mail: zlyynjzlk@163.com

${ }^{*}$ Contributed equally

Key words: pancreatic ductal adenocarcinoma, S100 calcium-binding protein A4, neutrophil-lymphocyte ratio, platelet-lymphocyte ratio, lymphocyte-monocyte ratio, prognostic factor (range, 1-32 months). Univariate analysis revealed that NLR, LMR, carbohydrate antigen 19-9, surgery, chemotherapy, stage at diagnosis, tumor grade and age significantly affected OS. Although PLR exhibited no significant effects on OS, NLR and LMR were independent prognostic factors according to the multivariate analysis. Unpaired Student's t-test revealed differences between S100A4 expression and NLR, PLR and LMR. The results of the present study indicated that low NLR and high LMR were associated with a favorable prognosis in patients with PDAC. As a simply obtained and widely available index at diagnosis, NLR and LMR may become a novel predictive and classifying marker for PDAC in the clinical setting.

\section{Introduction}

As one of the most fatal types of human malignant cancer, pancreatic ductal adenocarcinoma (PDAC) exhibits a poor prognosis, despite significant advances in diagnostic and therapeutic options, and has the lowest 5 year relative survival rate of $6 \%$ reported in 2016 in North America (1,2). Difficulties in detecting the disease at an early stage partially contribute to the poor prognosis (3). The search for novel biomarkers to detect and diagnose PDAC has been of interest to clinicians and researchers. Carbohydrate antigen 19-9 (CA19-9), the only authenticated marker for clinical application, lacks the specificity required for a differential diagnosis $(4,5)$. The majority of other markers are expensive or experimental, and are not widely used in routine clinical practice $(6,7)$. Therefore, there is an urgent need to identify convenient and easily applicable biomarkers for PDAC.

Peripheral blood examination is one of the most frequently used measures in tumor management. However, it is relatively rare to regard variables excluded from routine blood count parameters as prognostic factors (8). The neutrophil-to-lymphocyte ratio (NLR), platelet-to-lymphocyte ratio (PLR) and lymphocyte-to-monocyte ratio (LMR) at the initial diagnosis may serve as simple indexes of immune function, and each one is reported to be a prognostic factor in a number of different types of malignant tumor, such as Hodgkin's lymphoma, and bladder and hepatocellular cancer (9-12). 
However, their prognostic significance is controversial for patients with pancreatic cancer. Martin et al (13) investigated the effects of systemic inflammation-based factors, including NLR and PLR, on the outcome of patients with tumors, and concluded that both NLR and PLR were independent prognostic markers. However, Aliustaoglu et al (14) proposed that NLR was a superior marker for patients with pancreatic cancer. Furthermore, a previous study investigated the association between LMR and PDAC; one study indicated that low LMR predicted a poor prognosis in patients with resectable PDAC, but no further studies were pursued (15). Therefore, the prognostic value of the peripheral blood NLR, PLR and LMR in patients with PDAC was examined in the present study.

S100 calcium-binding protein A4 (S100A4), a member of the S100 family of calcium-binding proteins, promotes tumor metastasis, proliferation and immune evasion (16-19). A previous study has verified the hypothesis that S100A4-mediated metastasis is associated with extensive T-cell infiltration or tumor-associated neutrophils (20). S100A4 tissue expression was associated with a poor prognostic outcome in a variety of cancer types, including PDAC, and lung and breast cancer $(21,22)$. In the present study, the association between the pre-treatment peripheral blood NLR, PLR or LMR and S100A4 tissue expression was analyzed in 258 patients diagnosed with PDAC.

\section{Materials and methods}

Patient eligibility. The present study was conducted at the Tianjin Medical University Cancer Institute and Hospital (Tianjin, China). The study was approved by the Ethics Committee and all patients provided written informed consent. Patients with PDAC hospitalized between December 2008 and December 2014 were enrolled in the study. The clinical records of these patients were reviewed retrospectively. The inclusion criteria were: i) Patients were hospitalized for primary diagnosis and had received no treatment prior to diagnosis (therapy naïve); ii) patients were histologically diagnosed with primary PDAC and staged according to the Tumor-Node-Metastasis (TNM) criteria of the American Joint Committee on Cancer, 2017 (23); and iii) all clinical data for the patients were available. The exclusion criteria were: i) Patients did not have primary PDAC; ii) the detailed and required clinical data were unavailable; iii) patients had prior clinical evidence of infection, other inflammation, pulmonary embolism, acute myocardial infarction, cerebrovascular accident or hematological disease, or were taking drugs for hematological disorders; iv) patients had received prior radiation therapy, chemotherapy or surgery; and v) contact with the patients was lost during the follow-up time.

Clinical and laboratory data collection. Data regarding the patients, the tumor characteristics, the diagnosis and the treatment modalities were collected and retrospectively reviewed. In the current study, the majority of the complications were anastomotic leakage and ischemia-reperfusion; drug-controlled complications such as fever or abdominal infection were not included. For all study subjects, blood samples were collected at the first consultation in edathamil-2K preservative tubes, stored at room temperature and analyzed using the same hematology analyzer within $48 \mathrm{~h}$; differential leukocyte counts were recorded. The NLR, PLR and LMR were defined as the absolute neutrophil count divided by the absolute lymphocyte count, the absolute platelet count divided by the absolute lymphocyte count and the absolute lymphocyte count divided by the absolute monocyte count, respectively, in the laboratory tests prior to treatment. Tumor tissues and adjacent healthy tissues were collected during surgery or by $18-G$ needle biopsy.

Histopathological analysis. Tissues were fixed with $4 \%$ formalin at room temperature within $48 \mathrm{~h}$, embedded in paraffin, and diagnosed clinically and histopathologically at the Departments of Pancreatic Cancer and Pathology. All pathological data were analyzed by two pathologists independently.

Immunohistochemical (IHC) analysis was performed to evaluate the expression levels of S100A4 as previously described (24). Briefly, tissue sections with thickness of $3 \mu \mathrm{m}$ were incubated at $60^{\circ} \mathrm{C}$ for $2 \mathrm{~h}$ followed by deparaffinization with xylene and rehydration in concentrations of 100, 95, 85 and $75 \%$ alcohol, respectively. The sections were submerged in ethylenediamine tetraacetic acid antigen retrieval buffer (Beijing Solarbio Science \& Technology Co., Ltd.) and heated in a microwave for 2 min for antigen retrieval, treated with $3 \%$ hydrogen peroxide at room temperature for $10 \mathrm{~min}$ in methanol to quench endogenous peroxidase activity and incubated with $1 \%$ bovine serum albumin (Amresco LLC) to block non-specific binding. The sections were incubated with mouse anti-S100A4 (1:2,000; cat. no. ab197896; Abcam,) and diluent (cat. no. ZLI-9030; Origene Technologies, Inc.) overnight at $4^{\circ} \mathrm{C}$. Normal goat serum (Origene Technologies, Inc.) was used as a negative control. Tissues were subsequently incubated with the secondary antibody (cat. no. K183316C; Beijing Zhongshan Golden Bridge Biotechnology Co., Ltd.) at $37^{\circ} \mathrm{C}$ for $1 \mathrm{~h}$. Following 3 PBS washes, the tissue sections were counterstained with hematoxylin at room temperature for $3 \mathrm{~min}$, dehydrated and mounted.

The degree of IHC staining was reviewed and scored independently by two pathologists. IHC was scored by multiplying the scores of the percentage of positive tumor cells and staining intensity. The percentage of positive tumor cells was scored as $0(0 \%), 1(1-25 \%), 2(26-50 \%), 3(51-75 \%)$ or $4(76-100 \%)$. Staining intensity was scored as 0 (negative), 1 (weakly positive), 2 (moderately positive) or 3 (strongly positive). According to the staining results, the S100A4 tissue expression level was classified into two groups: Negative (score <3) and positive (score $\geq 3$ ).

Statistical analysis. Follow-up time was defined as the time between admission and August 2015. Overall survival (OS) time was defined as the interval between the time of diagnosis and final follow-up or death. Statistical analyses were performed using SPSS software version 21.0 (IBM Corp.). A $\chi^{2}$ test was performed to compare baseline clinical characteristics between patients of different subgroups. The survival curves were produced using Kaplan-Meier analysis. 
The log-rank and multivariate Cox proportional hazards regression model analyses were performed to determine the independent prognostic factors and survival function. The mean NLR, PLR and LMR data was compared for the subgroups with positive and negative S100A4 expression using an unpaired Student's t-test. X-tile analysis was used to identify the best cut-off value for low and high NLR, PLR and LMR. $\mathrm{P}<0.05$ was considered to indicate a statistically significant difference.

\section{Results}

Patient characteristics. Among the 258 patients with PDAC, the mean age was 59 years (median, 58.64 years; range, 21-75 years). The median OS time was 9 months (range, 1-32 months). A total of 105 patients were diagnosed based on the results of a biopsy, 70 patients received palliative surgery and 83 received radical surgery. Adjuvant chemotherapy and radiation therapy were performed following the biopsy or surgery. The median CA19-9 level was $260.45 \mathrm{U} / \mathrm{ml}$ (range, 0-100,254 U/ml). The clinicopathological characteristics of the patients and treatment modalities are presented in Table I.

Patient peripheral blood characteristics. At diagnosis, the median NLR, PLR and LMR were 2.55 (range, 0.63-19.00), 142.09 (range, 16.49-1,316.67) and 3.13 (range, 0.30-42.33), respectively. The baseline characteristics of the patients grouped by NLR, PLR or LMR quartiles are presented in Tables II-IV. The skewed frequency distribution of NLR, PLR and LMR is presented in Fig. 1, the majority of the patients exhibited NLR $<5$, PLR $<250$ and $\mathrm{LMR}<10$.

Patients in the highest NLR quartile were primarily male and had more pancreatic tumors of head and neck origin compared with the lowest quartile. In addition, the CA19-9 value was higher compared with that in the lowest NLR quartile (Table II). The patients in the highest PLR quartile had more pancreatic tumors of head and neck origin compared with those in the lowest PLR quartile. In addition, patients in the highest quartile were less likely to receive radiotherapy (Table III). The patients in the highest LMR quartile were primarily female compared with those in the lowest LMR quartile and no significant differences existed in the characteristics of the patients between the two groups (Table IV).

A significant increase was observed in OS among the patients in the lowest NLR quartile compared with those in the highest NLR quartile (median survival rate, 50.7 vs. $31.3 \%$, respectively; $\mathrm{P}<0.05$; Fig. 2A). No statistically significant difference was observed in the OS between patients in the lowest PRL quartile and those in the highest quartile (49.5 vs. $38.1 \%$, respectively; $\mathrm{P}>0.05$; Fig. $2 \mathrm{~B}$ ). By contrast, there was a significant decrease in OS between patients in the lowest LMR quartile compared with those with in the highest LMR quartile (28.3 vs. 57.8\%; P<0.05; Fig. 2C).

Risk factors of mortality. According to the univariate analysis, higher NLR, age, CA19-9 level, stage and histological grade were associated with a higher risk of mortality, whereas surgery, chemotherapy and higher LMR were associated with
Table I. Clinicopathological characteristics and treatment modalities in patients with pancreatic ductal adenocarcinoma.

\begin{tabular}{|c|c|c|}
\hline Characteristic & Number of patients & Percentage \\
\hline \multicolumn{3}{|l|}{ Sex } \\
\hline Male & 146 & 56.6 \\
\hline Female & 112 & 43.4 \\
\hline \multicolumn{3}{|c|}{ T stage at diagnosis } \\
\hline $\mathrm{T} 1$ & 1 & 0.4 \\
\hline $\mathrm{T} 2$ & 42 & 16.3 \\
\hline $\mathrm{T} 3$ & 118 & 45.7 \\
\hline $\mathrm{T} 4$ & 97 & 37.6 \\
\hline \multicolumn{3}{|c|}{$\mathrm{N}$ stage at diagnosis } \\
\hline No & 111 & 43.0 \\
\hline $\mathrm{N} 1$ & 119 & 46.1 \\
\hline $\mathrm{N} 2$ & 28 & 10.9 \\
\hline \multicolumn{3}{|c|}{ M stage at diagnosis } \\
\hline M0 & 165 & 64.0 \\
\hline M1 & 93 & 36.0 \\
\hline \multicolumn{3}{|c|}{ Stage at diagnosis } \\
\hline I & 16 & 6.2 \\
\hline II & 24 & 9.3 \\
\hline III & 125 & 48.5 \\
\hline IV & 93 & 36.0 \\
\hline \multicolumn{3}{|c|}{ Tumor differentiation } \\
\hline High & 26 & 10.1 \\
\hline Moderate & 109 & 42.2 \\
\hline Poor & 123 & 47.7 \\
\hline \multicolumn{3}{|l|}{ Tumor location } \\
\hline Head and neck & 165 & 64.0 \\
\hline Body and tail & 93 & 36.0 \\
\hline \multicolumn{3}{|c|}{ Adjuvant radiation therapy } \\
\hline Yes & 17 & 6.6 \\
\hline No & 241 & 93.4 \\
\hline \multicolumn{3}{|c|}{ Adjuvant chemotherapy } \\
\hline Yes & 219 & 84.9 \\
\hline No & 39 & 15.1 \\
\hline \multicolumn{3}{|l|}{ Complications } \\
\hline Yes & 20 & 7.8 \\
\hline No & 238 & 92.2 \\
\hline
\end{tabular}

lower mortality (Table V). The univariate analysis revealed that PLR had no significant effect on OS. The hazard ratio of mortality of patients with PDAC in the highest NLR quartile increased 1.765-fold $(\mathrm{P}=0.007)$ compared with those in the lowest. However, the hazard ratio of mortality of patients with PDAC in the highest LMR quartile decreased 0.501-fold $(\mathrm{P}=0.001)$ compared with those in the lowest LMR quartile (Table V).

Role of NLR, PLR and LMR as an independent predictor of mortality in PDAC. The variables associated with 
Table II. Baseline characteristics of patients with pancreatic ductal adenocarcinoma by neutrophil-to-lymphocyte ratio quartiles.

\begin{tabular}{llllll}
\hline Variable & $\mathrm{N}$ & 1st quartile & 2nd quartile & 3rd quartile & 4th quartile
\end{tabular}

\section{Sex}

Male

Female

$\mathrm{T}$ stage at diagnosis

$$
\mathrm{T} 1+\mathrm{T} 2
$$

T3

T4

$\mathrm{N}$ stage at diagnosis

$$
\text { N0 }
$$

$\mathrm{N} 2$

$\mathrm{M}$ stage at diagnosis

M0

M1

Stage at diagnosis

I

II

III

IV

Tumor differentiation

High
Moderate
Poor

Tumor location

Head and neck

Body and tail

Adjuvant radiation therapy

No

Yes

Surgery

None

Radical

Palliative

Adjuvant chemotherapy

None

GEM

GEM + others

Complications

No
Yes

CA19-9, U/ml

$<73.68$

73.68-260.45

$>260.45$ to $\leq 1,357$

$>1,357$
146

112

25

39

43

118

97

111

119

28

165

93

16

24

125

93

26

26
109

123

165

93

241

17

105

83

70

39

144

75

238

20

64

65

65

64
10

25

26

30

31

8

45

19

4

7

34

19

12

12
25

27

27

32

32

60

4

25

24

15

9

34

21

61

3

16

24

12

12

39

39
26

26

10

33

27

30

28

7

44

21

3

6

28

19

6

30

29

39

26

61

4

\section{4}

20

21

\section{8}

36

21

59

6

20

18

15

15
12

$0.008^{\mathrm{a}}$

44

21

0.816

$\begin{array}{rl}8 & 15 \\ 32 & 28 \\ 24 & 20\end{array}$

30

27

6

0.116

43

22

0.617

$\begin{array}{rr}4 & 5 \\ 6 & 5 \\ 36 & 27 \\ 23 & 23\end{array}$

0.212

$0.007^{\mathrm{a}}$

0.741

0.833

0.906

$12 \quad 10$

$\begin{array}{ll}37 & 37\end{array}$

$16 \quad 17$

0.735

$60 \quad 58$

56

$0.037^{\mathrm{a}}$

${ }^{\text {a }}<$ 0.05. CA19-9, carbohydrate antigen 19-9; GEM, gemcitabine.

NLR quartile and survival status in the Cox regression analyses were included in the Cox proportional hazard multivariate model, and all variables included in Table VI were associated with NLR quartile in previous analyses. The Cox proportional hazard multivariate analysis was performed separately to avoid combining NLR, PLR and 
Table III. Baseline characteristics of patients with pancreatic ductal adenocarcinoma by platelet-to-lymphocyte ratio quartiles.

\begin{tabular}{|c|c|c|c|c|c|c|}
\hline Variable & $\mathrm{N}$ & 1st quartile & 2nd quartile & 3rd quartile & 4th quartile & P-value \\
\hline Sex & & & & & & 0.318 \\
\hline Male & 146 & 38 & 42 & 34 & 32 & \\
\hline Female & 112 & 26 & 23 & 31 & 32 & \\
\hline $\mathrm{T}$ stage at diagnosis & & & & & & 0.764 \\
\hline $\mathrm{T} 1+\mathrm{T} 2$ & 43 & 10 & 10 & 10 & 13 & \\
\hline $\mathrm{T} 3$ & 118 & 27 & 28 & 33 & 30 & \\
\hline $\mathrm{T} 4$ & 97 & 26 & 30 & 21 & 20 & \\
\hline $\mathrm{N}$ stage at diagnosis & & & & & & 0.803 \\
\hline No & 111 & 27 & 28 & 27 & 29 & \\
\hline N1 & 119 & 30 & 29 & 35 & 25 & \\
\hline $\mathrm{N} 2$ & 28 & 7 & 6 & 9 & 8 & \\
\hline M stage at diagnosis & & & & & & 0.381 \\
\hline M0 & 165 & 41 & 38 & 47 & 39 & \\
\hline M1 & 93 & 23 & 27 & 18 & 25 & \\
\hline Stage at diagnosis & & & & & & 0.534 \\
\hline $\mathrm{I}$ & 16 & 5 & 4 & 4 & 3 & \\
\hline II & 24 & 7 & 7 & 6 & 4 & \\
\hline III & 125 & 32 & 30 & 29 & 34 & \\
\hline IV & 93 & 23 & 26 & 18 & 26 & \\
\hline Tumor differentiation & & & & & & 0.456 \\
\hline High & 26 & 7 & 3 & 9 & 7 & \\
\hline Moderate & 109 & 26 & 33 & 22 & 28 & \\
\hline Poor & 123 & 31 & 29 & 34 & 29 & \\
\hline Tumor location & & & & & & $0.007^{\mathrm{a}}$ \\
\hline Head and neck & 165 & 32 & 39 & 51 & 43 & \\
\hline Body and tail & 93 & 32 & 26 & 14 & 21 & \\
\hline Adjuvant radiation therapy & & & & & & $0.048^{\mathrm{a}}$ \\
\hline No & 241 & 57 & 64 & 63 & 57 & \\
\hline Yes & 17 & 7 & 1 & 2 & 7 & \\
\hline Surgery & & & & & & 0.457 \\
\hline None & 105 & 32 & 25 & 23 & 25 & \\
\hline Radical & 83 & 17 & 21 & 20 & 25 & \\
\hline Palliative & 70 & 15 & 19 & 22 & 14 & \\
\hline Adjuvant chemotherapy & & & & & & 0.173 \\
\hline None & 39 & 12 & 8 & 9 & 10 & \\
\hline GEM & 144 & 31 & 33 & 45 & 35 & \\
\hline GEM + others & 75 & 21 & 24 & 11 & 19 & \\
\hline Complications & & & & & & 0.940 \\
\hline No & 238 & 60 & 59 & 60 & 59 & \\
\hline Yes & 20 & 4 & 6 & 5 & 5 & \\
\hline CA19-9, U/ml & & & & & & 0.118 \\
\hline$<73.68$ & 64 & 14 & 18 & 14 & 18 & \\
\hline $73.68-260.45$ & 65 & 15 & 16 & 23 & 11 & \\
\hline$>260.45$ to $\leq 1,357$ & 65 & 19 & 9 & 16 & 21 & \\
\hline$>1,357$ & 64 & 16 & 22 & 12 & 14 & \\
\hline
\end{tabular}

${ }^{\text {a }}<0$ 05. CA19-9, carbohydrate antigen 19-9; GEM, gemcitabine.

LMR into one model, as they were highly associated with absolute lymphocyte counts. The results revealed that NLR was an independent predictor of mortality with a hazard ratio of $1.198(\mathrm{P}=0.017)$ as a continuous variable, whereas 1.543 as a categorical variable $(\mathrm{P}=0.058)$, therefore NLR cannot be used as an independent predictor of mortality as 
Table IV. Baseline characteristics of patients with pancreatic ductal adenocarcinoma by lymphocyte-to-monocyte ratio quartiles.

\begin{tabular}{llllll}
\hline Variable & $\mathrm{N}$ & 1st quartile & 2nd quartile & 3rd quartile & 4th quartile
\end{tabular}

\section{Sex}

Male

Female

$\mathrm{T}$ stage at diagnosis

$$
\begin{aligned}
& \mathrm{T} 1+\mathrm{T} 2 \\
& \mathrm{~T} 3
\end{aligned}
$$

$\mathrm{T} 4$

$\mathrm{N}$ stage at diagnosis

N0

$\mathrm{N} 2$

$\mathrm{M}$ stage at diagnosis

M0

M1

Stage at diagnosis

$\begin{array}{lr}\text { I } & 16 \\ \text { II } & 24 \\ \text { III } & 125 \\ \text { IV } & 93\end{array}$

Tumor differentiation

$\begin{array}{lr}\text { High } & 26 \\ \text { Moderate } & 109 \\ \text { Poor } & 123\end{array}$

Tumor location

Head and neck

Body and tail

Adjuvant radiation therapy

No
Yes

Surgery

None

Radical

Palliative

Adjuvant chemotherapy

None
GEM
GEM + others

Complications

No

Yes

CA19-9, U/ml

$<73.68$

$73.68-260.45$

$>260.45$ to $\leq 1,357$

$>1,357$
146

112

43

118

97

111

119

28

165

93

16

24

125

93

\section{6}

109

123

165

93

241

17

105

83

70

39

144

75

34

30

11

33

20

25

26

6

32

32

4

6

27

24

\section{3}

26

35

41

23

60

4

28

20

16

12

35

17

238

20

$64 \quad 17$

$65 \quad 7$

$65 \quad 19$

$64 \quad 21$

\section{7}

18

8

28

29

31

34

7

44

21

3
5
32
16

16

\section{6}

29

30

45

20

62

3

\section{5}

19

21

\section{0}

33

22

61

4

15

18

15

17

\section{0}

25

14

29

26

28

32

5

44

21

$\begin{array}{rr}6 & 3 \\ 7 & 6 \\ 29 & 27 \\ 29 & 24\end{array}$

6

32

27

37

28

61

4

24

24

17

10

37

18

60

5

18

20

16

11
25

39

10

28

22

27

27

10

45

19

0.062

0.347

6

27
24

11

22

31

0.225

0.521

22

58

6

0.741

28

20

16

7

39

18

60

4

14

20

15

15

${ }^{\mathrm{a}} \mathrm{P}<0.05$. CA19-9, carbohydrate antigen 19-9; GEM, gemcitabine.

a categorical variable. As a continuous variable, LMR was an independent predictor of mortality with a hazard ratio of $0.846(\mathrm{P}=0.021)$, but it was not a predictor as a categorical variable (hazard ratio, $0.663 ; \mathrm{P}=0.074$ ). PLR was not an independent predictor as a continuous or categorical variable (Table VI). 

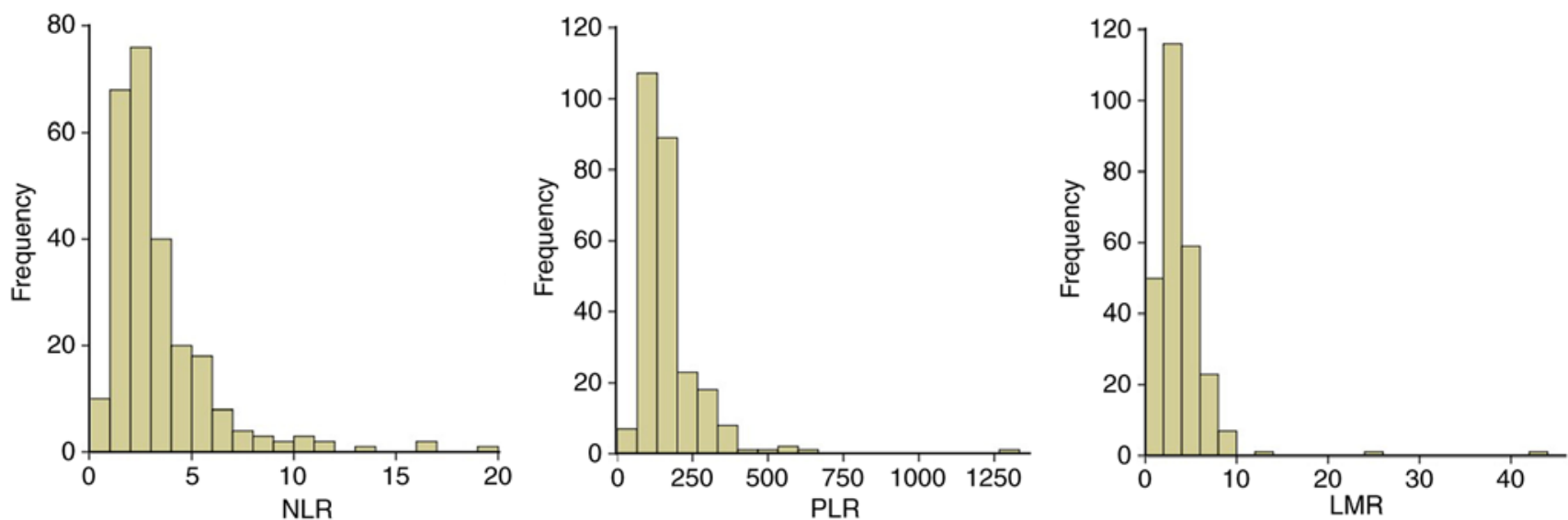

Figure 1. NLR, PLR and LMR distribution in 258 patients with pancreatic ductal adenocarcinoma. NLR, neutrophil-to-lymphocyte ratio; PLR, platelet-to-lymphocyte ratio; LMR, lymphocyte-to-monocyte ratio.
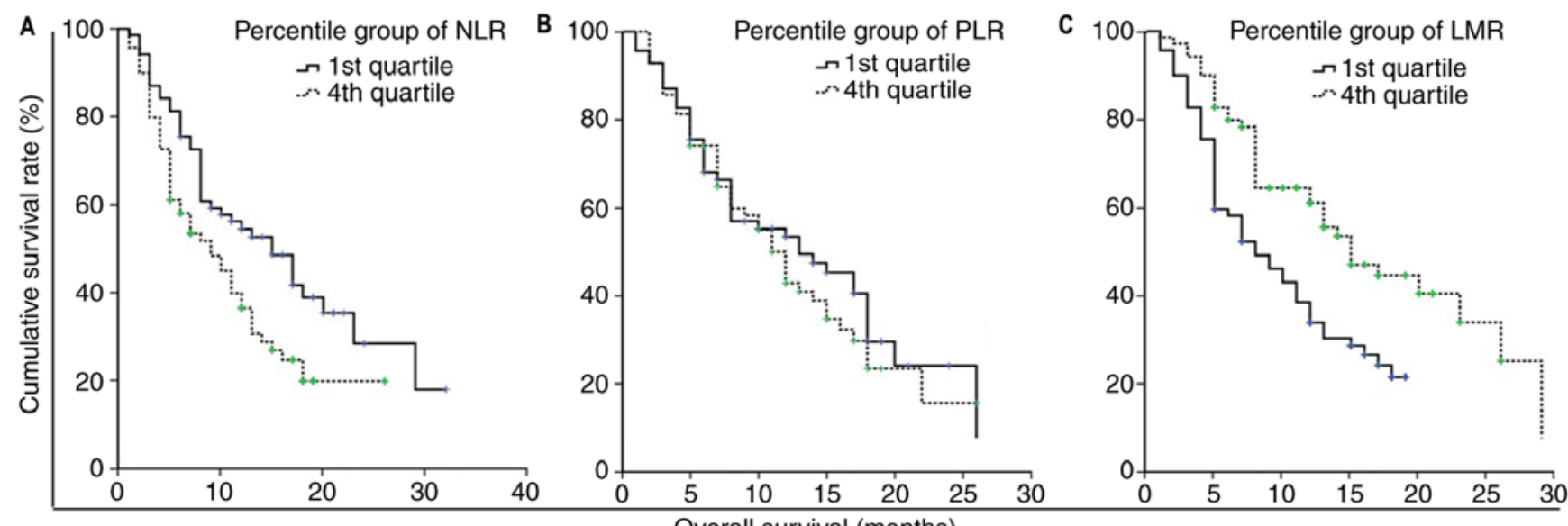

Figure 2. Kaplan-Meier survival curves based on different quartiles of (A) NLR, (B) PLR and (C) LMR in patients with pancreatic ductal adenocarcinoma. NLR, neutrophil-to-lymphocyte ratio; PLR, platelet-to-lymphocyte ratio; LMR, lymphocyte to monocyte ratio.

S100A4 expression and its association with peripheral blood NLR, PLR and LMR. The levels of peripheral blood NLR, PLR and LMR were analyzed in the subgroups of different expression levels of S100A4 (Fig. 3A). The number of patients with negative and positive tissue expression levels of S100A4 was 60 and 198, respectively. High expression levels of S100A4 were demonstrated to be associated with worse $\mathrm{OS}(\mathrm{P}=0.003$; Fig. 3B). The median value of NLR, PLR and LMR in the subgroup of negative S100A4 expression was 1.50, 114.19 and 4.70, respectively (Fig. 4). The mean value of NLR, PLR and LMR in the subgroup of positive S100A4 expression was 3.93 (median, 3.16), 181.10 (median, 152.45) and 3.46 (median, 2.73), respectively (Fig. 4). The levels of NLR and PLR in patients with positive S100A4 expression were higher compared with those in the negative S100A4 expression group $(\mathrm{P}<0.001$ and $\mathrm{P}=0.001$, respectively), whereas the level of LMR in patients with positive S100A4 expression was lower compared with that in the negative S100A4 expression group ( $\mathrm{P}=0.001$; Fig. 4).

Kaplan Meier survival analysis demonstrated better prognosis when NLR was lower than the cut-off value $(\mathrm{P}<0.001$; Fig. S1A). Furthermore, PLR had no significant effects on the prognosis $(\mathrm{P}>0.05$; Fig. $\mathrm{S} 1 \mathrm{~B})$. In contrast, LMR higher than the cut-off value predicted poor prognosis $(\mathrm{P}<0.001$; Fig. $\mathrm{S} 1 \mathrm{C})$.

\section{Discussion}

The aim of the present study was to identify simply obtained and inexpensive prognostic factors for PDAC. The prognostic significance of the peripheral blood NLR, PLR and LMR at diagnosis and their association with S100A4 expression in patients with PDAC were investigated. A number of studies have assessed the role of NLR in the outcome of PDAC and suggested that NLR may offer important prognostic information for the survival rate in patients with resectable PDAC $(6,25)$. In the present study, the prognostic role that NLR and LMR serve in PDAC was elucidated. Similarly to previous studies, a high NLR was an independent prognostic marker for the OS of patients with PDAC $(6,14,26)$. LMR has also been suggested to serve as a simple index of the immune function, and low LMR has been regarded as an independent predictor of poor prognosis in PDAC in a previous study (15). Consistent with the results of the previous study, the present study demonstrated that LMR possessed important prognostic information for PDAC and was associated with poor OS. According to studies by Kakkat et al (27) and Asari et al (28), high pre-treatment PLR is an independent predictive risk factor for patients with PDAC, which was not demonstrated in the 
Table V. Hazard ratios of baseline characteristics for mortality in patients with pancreatic ductal adenocarcinoma.

\begin{tabular}{|c|c|c|}
\hline Variable & Hazard ratio $(95 \% \mathrm{CI})$ & P-value \\
\hline Sex & $0.877(0.650-1.182)$ & 0.388 \\
\hline Age & $1.108(1.002-1.035)$ & $0.026^{\mathrm{a}}$ \\
\hline Stage at diagnosis (ref: $\mathrm{I}$ ) & & $<0.00^{\mathrm{a}}$ \\
\hline II & $0.431(0.219-0.969)$ & 1.512 \\
\hline III & $4.652(1.773-8.437)$ & $<0.001^{\mathrm{a}}$ \\
\hline IV & $3.273(1.728-6.202)$ & $<0.001^{\mathrm{a}}$ \\
\hline Tumor differentiation (ref: High) & & $<0.001^{\mathrm{a}}$ \\
\hline Moderate & $2.898(1.538-5.459)$ & $0.001^{\mathrm{a}}$ \\
\hline Poor & $5.524(2.900-10.522)$ & $<0.001^{\mathrm{a}}$ \\
\hline Tumor location & $1.140(0.839-1.551)$ & 0.402 \\
\hline Radiation therapy & $0.934(0.531-1.645)$ & 0.814 \\
\hline Complications & $0.737(0.426-1.275)$ & 0.275 \\
\hline Surgery (ref: None) & & $<0.001^{\mathrm{a}}$ \\
\hline Radical & $0.372(0.257-0.539)$ & $<0.001^{\mathrm{a}}$ \\
\hline Palliative & $0.688(0.483-0.979)$ & $0.038^{\mathrm{a}}$ \\
\hline Chemotherapy (ref: None) & & $0.037^{\mathrm{a}}$ \\
\hline GEM & $0.628(0.421-0.939)$ & $0.023^{\mathrm{a}}$ \\
\hline GEM + others & $0.579(0.371-0.901)$ & $0.016^{\mathrm{a}}$ \\
\hline CA19-9 (ref: $\leq 73.68)$ & & $0.004^{\mathrm{a}}$ \\
\hline$>73.68$ to 260.45 & $1.367(0.881-2.121)$ & 0.164 \\
\hline$>260.45$ to $\leq 1357$ & $1.494(0.970-2.300)$ & 0.068 \\
\hline$>1357$ & $2.163(1.419-3.297)$ & $<0.001^{\mathrm{a}}$ \\
\hline NLR quartile (ref: 1st) & & $0.009^{\mathrm{a}}$ \\
\hline 2nd & $0.945(0.612-1.459)$ & 0.797 \\
\hline $3 \mathrm{rd}$ & $1.414(0.925-2.161)$ & 0.109 \\
\hline 4 th & $1.765(1.164-2.676)$ & $0.007^{\mathrm{a}}$ \\
\hline PLR quartile (ref: 1st) & & 0.767 \\
\hline 2nd & $0.927(0.608-1.414)$ & 0.725 \\
\hline $3 \mathrm{rd}$ & $1.045(0.687-1.588)$ & 0.838 \\
\hline 4th & $1.156(0.762-1.753)$ & 0.495 \\
\hline LMR quartile (ref: 1st) & & $0.007^{\mathrm{a}}$ \\
\hline 2nd & $0.763(0.513-1.133)$ & 0.179 \\
\hline $3 \mathrm{rd}$ & $0.586(0.387-0.886)$ & $0.011^{\mathrm{a}}$ \\
\hline 4th & $0.501(0.328-0.765)$ & $0.001^{\mathrm{a}}$ \\
\hline
\end{tabular}

${ }^{a} \mathrm{P}<0.05$. Ref, reference; GEM, gemcitabine; CA19-9, carbohydrate antigen 19-9; NLR, neutrophil-to-lymphocyte ratio; PLR, platelet-to-lymphocyte ratio; LMR, lymphocyte-to-monocyte ratio.

present study. In addition, the majority of the patients received chemotherapy, but the effect of chemotherapy on overall survival was not investigated; the effect of chemotherapy on the statistical importance of NLR and PLR in OS needs to be further explored in the future.

S100A4 is involved in the proliferation, angiogenesis and invasion of tumor cells $(29,30)$. In the present study, it was revealed that patients with high S100A4 tissue expression exhibited unfavorable OS outcomes, which was similar to the results from previous studies (29-31). However, to the best of our knowledge, there are currently no studies that have been performed with the aim of evaluating the association between peripheral blood NLR, PLR and LMR and S100A4 expression. In the present study, NLR and PLR were positively associated with S100A4 expression, whereas LMR was negatively associated with S100A4 expression. The tumor microenvironment, comprising multiple cellular and molecular factors, serves a pivotal role in the biological behavior of numerous different types of cancer, including PDAC $(1,32)$. The microenvironment surrounding the tumor cells, containing cells of the immune system, is a prerequisite for regulating the initiation of metastasis and affects the prognosis of the malignancy $(32,33)$. The mechanism by which the microenvironment influences tumor metastasis is currently unknown, although it has been 
Table VI. Cox proportional multivariate hazard models in patients with pancreatic ductal adenocarcinoma.

A, Model A1 (NLR as a continuous variable)

\begin{tabular}{lcc}
\hline Variable & Hazard ratio (95\% CI) & P-value \\
\hline NLR & $1.198(1.033-1.389)$ & $0.017^{\mathrm{a}}$ \\
Age & $1.014(0.996-1.032)$ & 0.133 \\
Stage at diagnosis (ref: I) & & $0.012^{\mathrm{a}}$ \\
II & $1.199(0.583-2.465)$ & 0.622 \\
III & $2.968(1.382-6.056)$ & $0.004^{\mathrm{a}}$ \\
IV & $2.366(1.047-5.345)$ & $0.038^{\mathrm{a}}$ \\
Tumor differentiation & & $<0.001^{\mathrm{a}}$ \\
(ref: High) & & \\
Moderate & $2.248(1.175-4.299)$ & $0.014^{\mathrm{a}}$ \\
Poor & $3.942(2.004-7.752)$ & $<0.001^{\mathrm{a}}$ \\
Surgery (ref: None) & & $<0.001^{\mathrm{a}}$ \\
Radical & $0.578(0.335-0.997)$ & $0.049^{\mathrm{a}}$ \\
Palliative & $0.874(0.595-1.285)$ & 0.495 \\
Chemotherapy (ref: None) & & $0.037^{\mathrm{a}}$ \\
GEM & $0.682(0.433-1.073)$ & 0.098 \\
GEM + others & $0.650(0.402-1.052)$ & 0.080 \\
CA19-9 (ref: $\leq 73.68)$ & $1.373(0.849-2.221)$ & 0.197 \\
>73.68 to 260.45 & $1.249(0.802-1.944)$ & 0.326 \\
>260.45 to $\leq 1357$ & $1.503(0.967-2.337)$ & 0.070 \\
>1357 & & $0.004^{\mathrm{a}}$ \\
\hline
\end{tabular}

B, Model B1 (LMR as a continuous variable)

\begin{tabular}{lcc}
\hline Variable & Hazard ratio (95\% CI) & P-value \\
\hline LMR & $0.846(0.734-0.975)$ & $0.021^{\mathrm{a}}$ \\
Age & $1.012(0.994-1.031)$ & 0.181 \\
Stage at diagnosis (ref: I) & & $0.012^{\mathrm{a}}$ \\
II & $1.169(0.570-2.399)$ & 0.669 \\
III & $2.786(1.438-5.894)$ & $0.010^{\mathrm{a}}$ \\
IV & $2.214(0.985-4.975)$ & 0.054 \\
Tumor differentiation & & $<0.001^{\mathrm{a}}$ \\
(ref: High) & & \\
Moderate & $2.215(1.160-4.232)$ & $0.016^{\mathrm{a}}$ \\
Poor & $3.861(1.964-7.589)$ & $<0.001^{\mathrm{a}}$ \\
Surgery (ref: None) & & $<0.001^{\mathrm{a}}$ \\
Radical & $0.566(0.329-0.973)$ & $0.040^{\mathrm{a}}$ \\
Palliative & $0.855(0.581-1.260)$ & 0.429 \\
Chemotherapy (ref: None) & & $0.037^{\mathrm{a}}$ \\
GEM & $0.693(0.441-1.090)$ & 0.113 \\
GEM + others & $0.638(0.394-1.034)$ & 0.068 \\
CA19-9, U/ml (ref: $\leq 73.68)$ & $1.345(0.833-2.174)$ & 0.226 \\
$>73.68$ to 260.45 & $1.272(0.815-1.985)$ & 0.289 \\
$>$ >260.45 to $\leq 1,357$ & $1.504(0.965-2.345)$ & 0.071 \\
$>1,357$ & & $0.004^{\mathrm{a}}$ \\
& &
\end{tabular}

Table VI. Continued.

C, Model A2 (NLR as a categorical variable)

\begin{tabular}{lcc}
\hline Variable & Hazard ratio (95\% CI) & P-value \\
\hline NLR quartile (ref: 1st) & & $0.019^{\mathrm{a}}$ \\
2nd & $0.769(0.487-1.214)$ & 0.259 \\
3rd & $1.124(0.725-1.743)$ & 0.602 \\
4th & $1.543(0.986-2.415)$ & 0.058 \\
\hline
\end{tabular}

D, Model B2 (LMR as a categorical variable)

\begin{tabular}{lcc}
\hline Variable & Hazard ratio (95\% CI) & P-value \\
\hline LMR quartile (ref: 1st) & & 0.088 \\
2nd & $0.952(0.621-1.458)$ & 0.820 \\
3rd & $0.642(0.414-0.996)$ & $0.048^{\mathrm{a}}$ \\
4th & $0.663(0.422-1.040)$ & 0.074
\end{tabular}

${ }^{\text {aP }}<0.05$. Ref, reference; GEM, gemcitabine; CA19-9, carbohydrate antigen 19-9; NLR, neutrophil-to-lymphocyte ratio; LMR, lymphocyte-to-monocyte ratio.

suggested to be caused by S100A4 promoting tumor progression, metastasis and inflammation, either systemically or in the tumor microenvironment (34).

Certain studies focused on the genetic characteristics of the tumor $(35,36)$. However, a limited number of these prognostic models consider the role of host immunity (i.e., lymphocytes) and the microenvironment produced by the tumor (i.e., monocytes, neutrophils and S100A4) (15). In the present study, as well as NLR, peripheral blood LMR was revealed to serve a prognostic role in patients with PDAC. In addition, the association between peripheral blood NLR, PLR and LMR and the tissue expression of S100A4 was thoroughly analyzed in sufficient sample size. However, there were limitations to the present study, including the retrospective design, short follow-up periods and a relatively small sample size.

The present study provided evidence to support the prognostic use of NLR and LMR in patients with PDAC and demonstrated the prognostic relevance of host immunity and tumor-associated microenvironment when determining the clinical outcome. Further studies, including prospective clinical trials and mechanistic studies, are required in order to confirm the conclusions of the present study and reveal the underlying molecular mechanisms.

In conclusion, the present study demonstrated that in the peripheral blood from patients with PDAC, the highest NLR quartile and the lowest LMR quartile were associated with an unfavorable prognosis. The results of the present study also support the prognostic relevance of host immunity and the tumor-associated microenvironment when determining the clinical outcomes of patients with PDAC. As a simply obtained and widely available index at diagnosis, NLR and LMR may be a valid novel predictive and stratification marker for PDAC in clinical practice. 

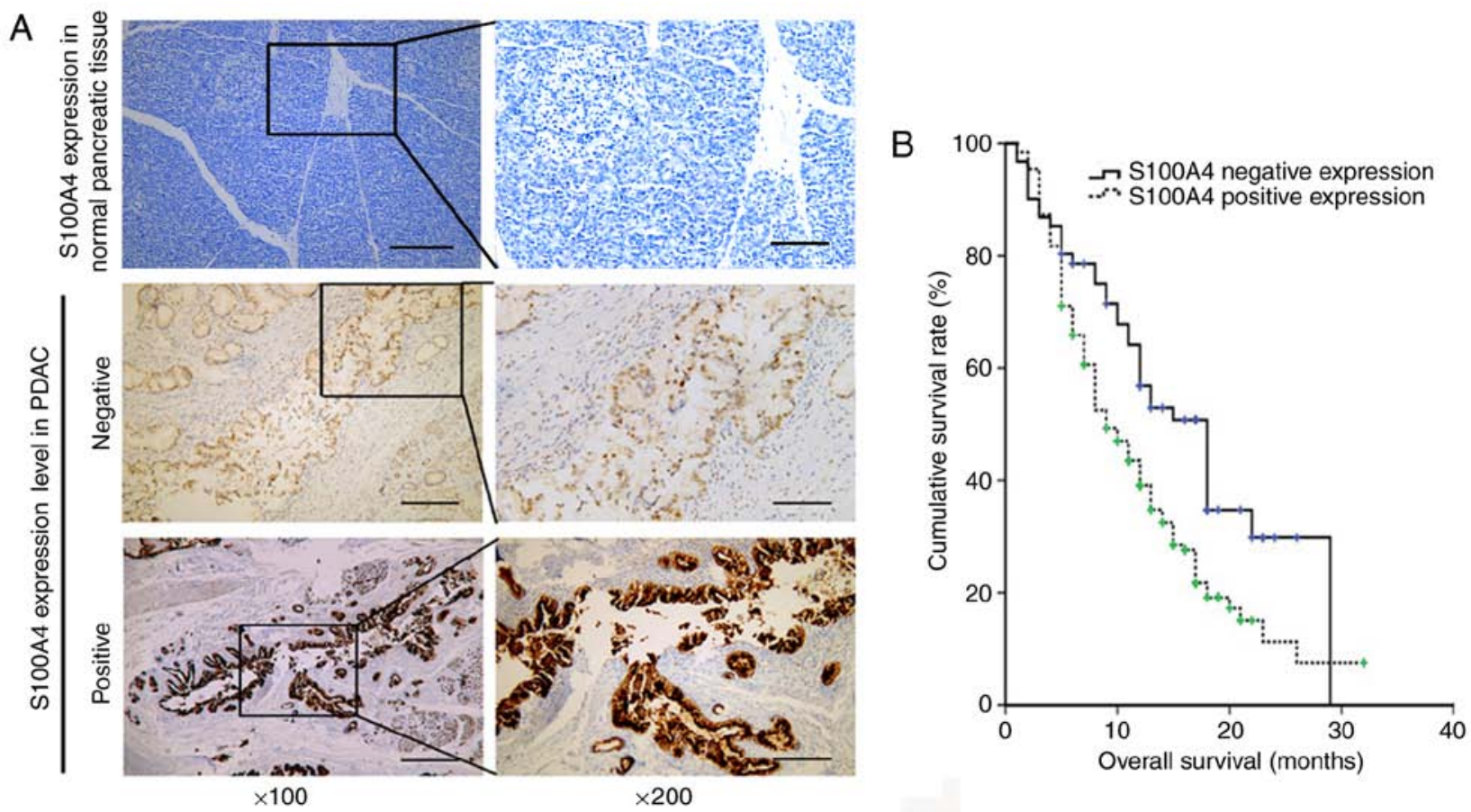

Figure 3. S100A4 expression in PDAC. (A) S100A4 tissue expression levels in normal pancreatic tissue and patients with PDAC. (B) Kaplan-Meier survival curves based on different expression levels of S100A4 in patients with PDAC. S100A4, S100 calcium-binding protein A4; PDAC, pancreatic ductal adenocarcinoma.
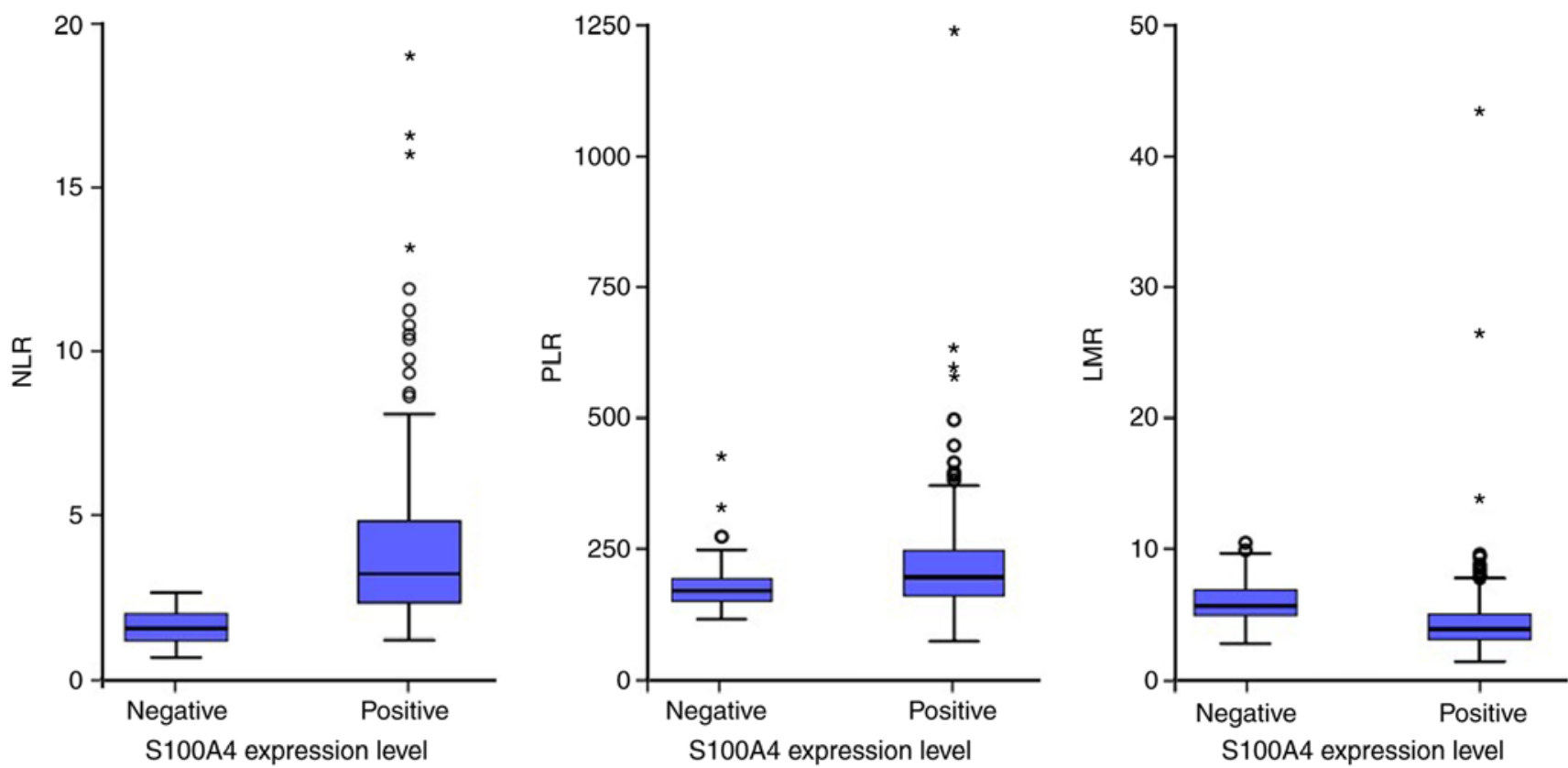

Figure 4. Comparison between peripheral blood NLR, PLR and LMR and positive and negative S100A4 expression. NLR, neutrophil-to-lymphocyte ratio; PLR, platelet-to-lymphocyte ratio; LMR, lymphocyte-to-monocyte ratio; S100A4, S100 calcium-binding protein A4.

\section{Acknowledgements}

Not applicable.

\section{Funding}

This study was funded by the Science and Technology Project of Tianjin colleges and Universities (grant no. 20130122), the Science Foundation of Tianjin Health Bureau (grant no. 2015KZ088), the Joint Funding Project of Tianjin Science Committee (grant no. 15JCYBJC49600) and the Scientific Research Foundation of Tianjin Medical University Cancer Institute and Hospital (grant no. 1706).

\section{Availability of data and materials}

All data generated or analyzed during this study are included in this published article. 


\section{Authors' contributions}

ZS and HL conceived and designed the study. HL and DZ designed the experiments. HL and XT performed the experiments and analyzed the data. YH obtained the epidemiological data. YX and YP performed the pathological analysis. HL and $\mathrm{XT}$ wrote, edited and revised the manuscript. All authors read and approved the final manuscript.

\section{Ethics approval and consent to participate}

The present study was approved by the Ethics Committee of the Tianjin Medical University Cancer Institute and Hospital (Tianjin, China), and all patients provided written informed consent.

\section{Patient consent for publication}

Not applicable.

\section{Competing interests}

The authors declare that they have no competing interests.

\section{References}

1. Foucher ED, Ghigo C, Chouaib S, Galon J, Iovanna J and Olive D: Pancreatic ductal adenocarcinoma: A strong imbalance of good and bad immunological cops in the tumor microenvironment. Front Immunol 9: 1044, 2018.

2. Zheng L, Xue J, Jaffee EM and Habtezion A: Role of immune cells and immune-based therapies in pancreatitis and pancreatic ductal adenocarcinoma. Gastroenterology 144: 1230-1240, 2013.

3. Shi C, Merchant N, Newsome G, Goldenberg DM and Gold DV: Differentiation of pancreatic ductal adenocarcinoma from chronic pancreatitis by PAM4 immunohistochemistry. Archives Pathol Lab Med 138: 220-228, 2014.

4. Hernandez JM, Cowgill SM, Al-Saadi S, Collins A, Ross SB, Cooper J, Villadolid D, Zervos E and Rosemurgy A: CA 19-9 velocity predicts disease-free survival and overall survival after pancreatectomy of curative intent. J Gastrointest Surg 13: 349-353, 2009

5. Winter JM, Yeo CJ and Brody JR: Diagnostic, prognostic, and predictive biomarkers in pancreatic cancer. J Surg Oncol 107: $15-22,2013$.

6. Hanahan D and Weinberg RA: Hallmarks of cancer: The next generation. Cell 144: 646-674, 2011

7. Marengo E and Robotti E: Biomarkers for pancreatic cancer: Recent achievements in proteomics and genomics through classical and multivariate statistical methods. World J Gastroenterol 20: 13325-13342, 2014.

8. Roxburgh CS and McMillan DC: Role of systemic inflammatory response in predicting survival in patients with primary operable cancer. Future Oncol 6: 149-163, 2010.

9. Koh YW, Kang HJ, Park C, Yoon DH, Kim S, Suh C, Go H, Kim JE, Kim CW and Huh J: The ratio of the absolute lymphocyte count to the absolute monocyte count is associated with prognosis in hodgkin's lymphoma: Correlation with tumor-associated macrophages. Oncologist 17: 871-880, 2012.

10. Kaynar M, Yildirim ME, Badem H, Cavis M, Tekinarslan E, Istanbulluoglu MO, Karatas OF and Cimentepe E: Bladder cancer invasion predictability based on preoperative neutrophil-lymphocyte ratio. Tumour Biol 35: 6601-6605, 2014.

11. Tanoglu A and Karagoz E: Neutrophil and platelet-to-lymphocyte ratio: New predictors of dropout and recurrence after liver transplantation for hepatocellular cancer? Transplant Int 27: e80-e81, 2014.

12. McMillan DC: Systemic inflammation, nutritional status and survival in patients with cancer. Curr Opin Clin Nutr Metab Care 12: 223-226, 2009.
13. Martin HL, Ohara K, Kiberu A, Van Hagen T, Davidson A and Khattak MA: Prognostic value of systemic inflammation-based markers in advanced pancreatic cancer. Intern Med J 44: 676-682, 2014.

14. Aliustaoglu M, Bilici A, Seker M, Dane F, Gocun M, Konya V, Ustaalioglu BB and Gumus M: The association of pre-treatment peripheral blood markers with survival in patients with pancreatic cancer. Hepatogastroenterology 57: 640-645, 2010.

15. Sierzega M, Lenart M, Rutkowska M, Surman M, Mytar B, Matyja A, Siedlar M and Kulig J: Preoperative neutrophil-lymphocyte and lymphocyte-monocyte ratios reflect immune cell population rearrangement in resectable pancreatic cancer. Ann Surg Oncol 24: 808-815, 2017.

16. Garrett SC, Varney KM, Weber DJ and Bresnick AR: S100A4, a mediator of metastasis. J Biol Chem 281: 677-680, 2006.

17. Liang J, Piao Y, Holmes L, Fuller GN, Henry V, Tiao N and de Groot JF: Neutrophils promote the malignant glioma phenotype through S100A4. Clin Cancer Res 20: 187-198, 2014.

18. Hansen MT, Forst B, Cremers N, Quagliata L, Ambartsumian N, Grum-Schwensen B, Klingelhofer J, Abdul-Al A, Herrmann P, Osterland M, et al: A link between inflammation and metastasis: Serum amyloid A1 and A3 induce metastasis, and are targets of metastasis-inducing S100A4. Oncogene 34: 424-435, 2015.

19. Dahlmann M, Kobelt D, Walther W, Mudduluru G and Stein U: S100A4 in cancer metastasis: Wnt signaling-driven interventions for metastasis restriction. Cancers (Basel) 8: E59, 2016.

20. Grum-Schwensen B, Klingelhofer J, Grigorian M, Almholt K, Nielsen BS, Lukanidin E and Ambartsumian N: Lung metastasis fails in MMTV-PyMT oncomice lacking S100A4 due to a T-cell deficiency in primary tumors. Cancer Res 70: 936-947, 2010.

21. Yao R, Davidson DD, Lopez-Beltran A, MacLennan GT, Montironi R and Cheng L: The S100 proteins for screening and prognostic grading of bladder cancer. Histol Histopathol 22: 1025-1032, 2007

22. Ai KX, Lu LY, Huang XY, Chen W and Zhang HZ: Prognostic significance of S100A4 and vascular endothelial growth factor expression in pancreatic cancer. World J Gastroenterol 14: 1931-1935, 2008.

23. Kamarajah SK, Burns WR, Frankel TL, Cho CS and Nathan H: Validation of the american joint commission on cancer (AJCC) 8th edition staging system for patients with pancreatic adenocarcinoma: A surveillance, epidemiology and end results (SEER) analysis. Ann Surg Oncol 24: 2023-2030, 2017.

24. Tian X, Zhou D, Chen L, Tian Y, Zhong B, Cao Y, Dong Q, Zhou M, Yan J, Wang Y, et al: Polo-like kinase 4 mediates epithelial-mesenchymal transition in neuroblastoma via PI3K/Akt signaling pathway. Cell Death Dis 9: 54, 2018.

25. Garcea G, Ladwa N, Neal CP, Metcalfe MS, Dennison AR and Berry DP: Preoperative neutrophil-to-lymphocyte ratio (NLR) is associated with reduced disease-free survival following curative resection of pancreatic adenocarcinoma. World J Surg 35: 868-872, 2011.

26. Smith RA, Bosonnet L, Raraty M, Sutton R, Neoptolemos JP, Campbell F and Ghaneh P: Preoperative platelet-lymphocyte ratio is an independent significant prognostic marker in resected pancreatic ductal adenocarcinoma. Am J Surg 197: 466-472, 2009.

27. Kakkat S, Rajan R, Sindhu RS, Natesh B and Raviram S: Comparison of platelet-lymphocyte ratio and CA 19-9 in differentiating benign from malignant head masses in patients with chronic pancreatitis. Indian J Gastroenterol 36: 263-267, 2017.

28. Asari S, Matsumoto I, Toyama H, Shinzeki M, Goto T, Ishida J, Ajiki T, Fukumoto T and Ku Y: Preoperative independent prognostic factors in patients with borderline resectable pancreatic ductal adenocarcinoma following curative resection: The neutrophil-lymphocyte and platelet-lymphocyte ratios. Surg Today 46: 583-592, 2016.

29. Jia W, Gao XJ, Zhang ZD, Yang ZX and Zhang G: S100A4 silencing suppresses proliferation, angiogenesis and invasion of thyroid cancer cells through downregulation of MMP-9 and VEGF. Eur Rev Med Pharmacol Sci 17: 1495-1508, 2013.

30. Tsukamoto N, Egawa S, Akada M, Abe K, Saiki Y, Kaneko N, Yokoyama S, Shima K, Yamamura A, Motoi F, et al: The expression of S100A4 in human pancreatic cancer is associated with invasion. Pancreas 42: 1027-1033, 2013.

31. Mahon PC, Baril P, Bhakta V, Chelala C, Caulee K, Harada T and Lemoine NR: S100A4 contributes to the suppression of BNIP3 expression, chemoresistance, and inhibition of apoptosis in pancreatic cancer. Cancer Res 67: 6786-6795, 2007.

32. Lee SH, Kim H, Hwang JH, Shin E, Lee HS, Hwang DW, Cho JY, Yoon YS, Han HS and Cha BH: CD24 and S100A4 expression in resectable pancreatic cancers with earlier disease recurrence and poor survival. Pancreas 43: 380-388, 2014. 
33. Arumugam T, Ramachandran V, Gomez SB, Schmidt AM and Logsdon CD: S100P-derived RAGE antagonistic peptide reduces tumor growth and metastasis. Clin Cancer Res 18: 4356-4364, 2012.

34. Langley RR and Fidler IJ: The seed and soil hypothesis revisited-the role of tumor-stroma interactions in metastasis to different organs. Int J Cancer 128: 2527-2535, 2011.

35. Knudsen ES, Vail P, Balaji U, Ngo H, Botros IW, Makarov V, Riaz N, Balachandran V, Leach S, Thompson DM, et al: Stratification of pancreatic ductal adenocarcinoma: Combinatorial genetic, stromal, and immunological markers. Clin Cancer Res 23: 4429-4440, 2017.
36. Raphael BJ, Hruban RH, Aguirre AJ, Moffitt RA, Yeh JJ, Stewart C, Robertson AG, Cherniack AD, Gupta M, Getz G, et al: Integrated genomic characterization of pancreatic ductal adenocarcinoma. Cancer Cell 14: 185-203, 2017.

This work is licensed under a Creative Commons Attribution-NonCommercial-NoDerivatives 4.0 International (CC BY-NC-ND 4.0) License. 